\title{
1 Model based Prediction of Nanostructured Thin Film Morphology in an Aerosol Chemical Vapor Deposition Process
}

\author{
3 Tandeep S. Chadha ${ }^{\mathrm{a}}$,Mengmeng Yang ${ }^{\mathrm{a}, \mathrm{b}}$, Kelsey Haddad ${ }^{\mathrm{a}}$, Vivek B. Shah ${ }^{\mathrm{a}}$, Shuiqing $\mathrm{Li}^{\mathrm{b}}$, \\ Pratim Biswas ${ }^{\mathrm{a}, *}$ \\ 5 \\ ${ }^{a}$ Aerosol and Air Quality Research Laboratory \\ Washington University in St. Louis \\ Department of Energy, Environmental \& Chemical Engineering \\ St. Louis, MO-63130, USA \\ ${ }^{\mathrm{b}}$ Key Laboratory for Thermal Science and Power Engineering of Ministry of Education, \\ Department of Thermal Engineering \\ Tsinghua University \\ Beijing, China \\ To be Submitted to : \\ $20 *$ To whom correspondence should be addressed: \\ 21 Tel: +1-314-935-5548; Fax: +1-314-935-5464 \\ 22 E-mail address: pbiswas@wustl.edu
}




\section{Abstract}

24 The aerosol chemical vapor deposition (ACVD) process has been demonstrated as a promising 25 approach to the single step synthesis of nanostructured metal oxide thin films. Multiple process 26 parameters control the nanostructure morphology and the growth of thin films. This work

27 focuses on utilizing a simulation based approach to understand the role of these parameters in 28 governing the morphology of the thin film. A finite element based computational fluid dynamics 29 model, coupled with a discrete-sectional aerosol model, and a boundary layer diffusion and 30 sintering model has been formulated to predict the evolution of particle size distribution and the 31 morphology of the synthesized nanostructured film. The morphology predicted by the model was

32 validated by experimental observations. The model enables scale up and wider application of the 33 ACVD process and can be extended to other gas phase deposition systems.

34 Keywords: Nanostructures, Chemical vapor deposition, Computational fluid dynamics, 35 Modeling, Aerosol size distribution, Sintering 


\section{$38 \quad 1 \quad$ Introduction}

39 Nanostructured thin films synthesis has attracted enormous interest in the past several decades

40 due to their wide array of applications including solar photovoltaics[2-4], solar water-splitting [1,

41 5-11], $\mathrm{CO}_{2}$ photoreduction [12, 13], and lithium-ion batteries [14]. Various approaches have

42 been used to synthesize these nanostructured films, such as, thermal oxidation [15], vapor-liquid

43 solid (VLS) growth [16], template-based synthesis [17], electrochemical methods [18] and gas

44 phase methods [1, 19, 20]. Among these methods, gas phase synthesis such as flame aerosol

45 deposition [19], particle-precipitation chemical vapor deposition (PP-CVD) [21], and aerosol

46 chemical vapor deposition (ACVD) [22] present a rapid, scalable and low-cost method of

47 synthesizing nanostructured thin films. These gas phase methods utilize the direct deposition and

48 sintering of particles onto substrates to form the desired thin film morphology. Three

49 predominant morphologies, namely, dense, columnar, and granular have been reported in the

50 literature [1, 2, 23]. For applications in solar energy and energy storage, the columnar

51 morphology has proven to be the most advantageous due to its oriented, single crystalline, one-

52 dimensional structure, high surface area, and fast electron transfer[1, 2, 6, 14].

53 Increasing interest in the nanostructured thin films synthesized by the ACVD process and desire

54 to scale-up the process for commercial application has prompted further research into

55 understanding the effect of process parameters on the aerosol dynamics in the reactor and the

56 synthesized morphology. While, the process is simple to operate, the intricate combination of

57 fluid dynamics, heat transfer and aerosol dynamics needed to achieve control over the

58 synthesized morphology is relatively complex. Understanding the relationship between process

59 parameters and the morphology is crucial for scalability and commercial implementation of these

60 processes. Efforts have been made to understand the factors that govern the morphology in such 
61 processes. Thimsen and Biswas [19] studied the effect of precursor feed rate and the substrate

62 temperature on the morphology, crystalline nature and photoactive performance of

63 nanostructured $\mathrm{TiO}_{2}$ thin films synthesized by a flame aerosol deposition process. The two

64 parameters were linked to four characteristic times, the residence time $\left(\tau_{\text {res }}\right)$, the reaction time

$65\left(\tau_{r x n}\right)$, the collision time $\left(\tau_{c o l}\right)$ and the sintering time $\left(\tau_{s i n}\right)$. Based on a qualitative comparison

66 between these four characteristic times, three regimes in the deposition process were identified

67 namely, chemical vapor deposition (CVD), individual particle deposition (IPD) and agglomerate

68 particle deposition (APD), which yielded the three different morphologies stated above. An et.

69 al. [1] utilized a similar empirical approach for understanding the link between the process

70 parameters and the morphology in the aerosol chemical vapor deposition process (ACVD)

71 utilizing the same characteristic times to identify three regimes — vapor only, vapor-particle mix

72 and particle only regime. However, for the ACVD process, the process parameters often

73 influence more than one characteristic time, making it difficult to correlate to the morphology of

74 the synthesized films to characteristic times. As an example, increasing the substrate temperature

75 leads to a decrease in $\tau_{r x n}, \tau_{c o l}$, and $\tau_{s i n}$ but the effect on morphology would be dictated by the

76 relative magnitude of decrease in each of these times, which cannot be determined by these

77 qualitative approaches. This warrants the need for a quantitative approach, accounting for both

78 the computational fluid dynamics (CFD) and the aerosol dynamics in the process.

79 Numerous studies have focused on coupling aerosol dynamics with CFD simulations for

80 aerosol reactors [24, 25]. In order to do this coupling in a computationally efficient manner,

81 approximations are often made on the CFD model and/or the aerosol dynamics model. Various

82 aerosol dynamics models such as monodisperse, lognormal [26], modal [27], sectional [28], and

83 discrete-sectional [29] have been developed to simulate aerosol dynamics in gas phase 
84 reactors[30], as well as in atmospheric processes [31]. The selection of the aerosol dynamics

85 model and the coupling depends on a number of factors that characterize the system being 86 modeled. Grohen et. al. [32] and Schild et al. [24] applied mono-disperse aerosol dynamics

87 models interfacing with the fluid mechanics to simulate the particle production in a flame spray

88 pyrolysis reactor and a premixed aerosol reactor, respectively. The mono-disperse assumption

89 reduces the number of aerosol dynamics equations on each discretized node to one, which

90 substantially lowers the computational requirement. However, this simplification leads to

91 inaccuracies in the particle size distribution. Prior literature for the ACVD process suggests that

92 the particles depositing on the substrate are smaller than $5 \mathrm{~nm}$. This makes the particle size

93 information extremely critical and higher accuracies are desired, especially in the lower size

94 range close to the nucleation size $(0.49 \mathrm{~nm})$.

95 In order to address the issue of computational time and desired accuracies in the aerosol size 96 distribution, this work utilizes a COMSOL ${ }^{\circledR}$ multiphysics finite element simulation coupled one 97 way to an aerosol dynamics model to simulate particle formation and growth in the reactor. The 98 discrete sectional model (DSM) is chosen for the simulation of aerosol dynamics due to its 99 molecular size resolution in the lower size range and the computational efficiency in the higher 100 size range. Finally, the particle size distribution arriving at the boundary layer of the deposition 101 substrate is correlated to the nanostructured morphology by simulating Brownian diffusion in the 102 boundary layer and sintering on the substrate. The findings from the simulation have been 103 validated by experimental results reported by An et. al [1] . The influence of process conditions 104 on the particle size distribution and the resulting film morphology has been reported and an 105 understanding of the governing parameters is developed. 


\section{$106 \quad 2$ Methods}

\section{$107 \quad 2.1 \quad$ Experimental process description}

108 The ACVD process is specifically designed for the synthesis of nanostructured thin films while 109 maintaining precise control over the morphology and the crystallinity of the thin films. The 110 process originates from the flame aerosol deposition reactor but, it exhibits certain distinguishing

111 features which will be described later. The process (Figure 1a) is comprised of three parts: the 112 precursor feed system, the reaction chamber, and the control system. The precursor feed system

113 contains a volatile precursor in a bubbler maintained at a constant temperature. Carrier gas $\left(\mathrm{N}_{2}\right)$

114 is bubbled through the volatile precursor to feed the precursor vapors into the reactor system via 115 a feeder tube accompanied by an optional dilution system. The precursor reaction and aerosol 116 dynamics occur in the reaction chamber, maintained at atmospheric pressure, as a result of the 117 heat flux from the heated substrate. The aerosol is transported via mass convection to the heated 118 substrate,where the vapor-particle mix is then deposited through Brownian diffusion in the 119 boundary layer above the substrate. On the substrate, the vapors react and the particles sinter to 120 form nanostructured thin films.

121 The control system provides real-time control over various process parameters in the precursor

122 feed system and the reaction chamber, leading to consistent and reliable operation of the process.

123 These process parameters include the carrier gas flow rate $\left(\mathrm{Q}_{1}\right)$, the precursor temperature $\left(\mathrm{T}_{\mathrm{wb}}\right)$, 124 the dilution gas flow rate $\left(\mathrm{Q}_{2}\right)$, and the dilution gas temperature $\left(\mathrm{T}_{2}\right)$ in the precursor feed system. 125 For the modeling study, the above process parameters can be translated into three parameters 126 namely, the initial precursor concentration $\left(\mathrm{c}_{0}\right)$, the total flow rate $\left(\mathrm{Q}_{0}\right)$, and the initial precursor 127 feed flow temperature $\left(\mathrm{T}_{0}\right)$. In the reaction chamber, the process parameters controlled are the 
128 substrate temperature $\left(\mathrm{T}_{\mathrm{s} 0}\right)$ and the feeder tube-substrate distance $\left(\mathrm{h}_{0}\right)$. In the present work, we

129 investigate the influence of these five parameters $\left(c_{0}, Q_{0}, T_{0}, T_{s 0}\right.$, and $\left.h_{0}\right)$ on the aerosol particle

130 size distribution and the morphology of the synthesized nanostructured thin film. In most cases,

131 since the columnar morphology is desired, the process parameters reported by An et al. for

132 columnar morphology growth is chosen as the reference case (base set of parameters) and the

133 effect of each parameter is studied[1]. The details of these simulation cases are listed in Table $\mathbf{1}$.

134 Although, the system has previously been used to synthesize thin films of various metal-oxides,

135 the present work focuses on the synthesis of $\mathrm{TiO}_{2}$ using titanium tetraisopropoxide (TTIP) as the

136 precursor.

$137 \quad 2.2 \quad$ Simulation of temperature and fluid flow

138 The fluid dynamics and heat transfer simulation, together with the mass transport are 139 implemented using COMSOL ${ }^{\circledR}$, a commercial finite element software designed for solving 140 problems of multi-physics. The simulation domain is shown in Figure $1 \mathrm{~b}$ simulated as a 2D 141 axisymmetric geometry in COMSOL ${ }^{\circledR}$. The equations for the heat transfer in the reactor 142 components and the fluid phase along with the fluid flow and the mass transport of the vapor 143 species are solved simultaneously at steady state using the heat transfer, laminar flow, and 144 transport of diluted species physics in the package. The simulation zone is discretized using a 145 free triangular mesh with a finer mesh for the zone between the end of the feeder tube and the 146 substrate (defined as the refined zone). Corner and boundary layer refinement of the mesh is 147 carried out for regions close to any surface. A laminar inflow boundary condition for the flow is 148 used at the inlet of the reactor and a constant pressure boundary condition is used for the outflow. 149 A no-slip boundary condition is applied to the flow interaction with the substrate. A convective 
150 cooling boundary condition with external natural convection is applied to the outside wall of the 151 glass chamber.

152 Outputs of the finite element simulation, including the velocity profile, the temperature profile

153 and the reference vapor concentration are utilized as inputs to the aerosol dynamics model. This

154 one-way coupling can be done under the assumption that the aerosols do not affect the fluid flow

155 or the temperature profile within the reaction chamber. Further, since the Peclet number for

156 particle transport is considerably high, in the range of 20 to $10^{5}$ for particle sizes ranging from

1570.4 to $20 \mathrm{~nm}$ in the temperature range under consideration, the diffusion of particles in the

158 reaction chamber has been ignored. The discrete sectional model provides the solution for the

159 temporal evolution of the aerosol size distribution and is performed using a Lagrangian approach

160 along the velocity streamlines obtained from the CFD simulation. The simulation is done on

161 different streamlines at varying radial distance from the axisymmetric axis upto the entrance of

162 the substrate boundary layer, which is defined as the region above the substrate at which the

163 radial velocity of the flow reaches $99 \%$ of the velocity magnitude[33]. The Peclet number for

164 particle transport in the boundary layer tends to 0 and hence the above simulation scheme can no

165 longer be applied. Instead, a different simulation scheme that only considers Brownian diffusion

166 should be implemented to describe the boundary layer.

\section{$167 \quad 2.3 \quad$ Implementation of aerosol dynamics}

168 Various mechanisms participate in the particle size evolution, including the precursor 169 decomposition, vapor nucleation, condensation, coagulation and surface growth. The aerosol 170 dynamics simulated and the modeling approach for film morphology prediction is shown in

171 Figure 2. These mechanisms are all considered in the aerosol dynamics equation [34]: 
172

$$
\begin{aligned}
\frac{\partial n}{\partial t}+\frac{\partial(G n)}{\partial v}+\frac{\partial(S n)}{\partial v}-I\left(v^{*}\right) \delta\left(v-v^{*}\right) & \\
& =\frac{1}{2} \int_{0}^{v} \beta(v-u, u) n(v-u, t) n(u, t) d u-n(v, t) \int_{0}^{\infty} \beta(v, u) n(u, t) d u
\end{aligned}
$$

173 wheren is the particle size distribution function, a function of particle size and time $(t)$. The latter

174 three terms on the left in Equation (1) represent the processes of condensation, surface growth, 175 and nucleation respectively, while terms on the right side account for the coagulation process 176 where $\beta(v, u)$ is the kinetic coefficient of coagulation of two particles of volume $v$ and $u$. For $177 \mathrm{TiO}_{2}$ particles originating from the thermal decomposition of TTIP, the molecular volume of $178 \mathrm{TiO}_{2}\left(62.70 \AA^{3}\right)$ exceeds the critical volume for nucleation $\left(31.42 \AA^{3}\right)$ and hence the formation 179 rate of monomers can be directly calculated by the precursor decomposition rate, expressed 180 by[35]:

$$
R_{g}=k_{g} C_{T T I P} ; k_{g}=1.54 \times 10^{6}\left[\mathrm{~s}^{-1}\right] \exp \left(-\frac{74.03\left[\frac{\mathrm{kJ}}{\mathrm{mol}}\right]}{R T}\right)
$$

181 where $R_{\mathrm{g}}\left[\mathrm{mol} \cdot \mathrm{m}^{-3} \mathrm{~s}^{-1}\right]$ is the TTIP decomposition rate, $k_{\mathrm{g}}$ is the reaction rate constant and $C_{\text {TTIP }}$ $182\left[\mathrm{~mol} . \mathrm{m}^{-3}\right]$ is the concentration of the TTIP precursor in the gas phase. Surface growth is dictated 183 by the reaction of TTIP on the $\mathrm{TiO}_{2}$ surface, and the surface growth rate is expressed by[36]:

$$
R_{S}=k_{S} A C_{T T I P} ; k_{S} \approx 1 \times 10^{11}\left[\mathrm{~cm} \cdot \mathrm{s}^{-1}\right] \exp \left(-\frac{15155.16[\mathrm{~K}]}{T}\right)
$$

185 where $R_{\mathrm{s}}\left[\mathrm{mol} \cdot \mathrm{m}^{-3} \mathrm{~s}^{-1}\right]$ and $k_{\mathrm{s}}$ are the surface growth rate and reaction constant respectively, and A $186\left[\mathrm{~cm}^{2} \cdot \mathrm{cm}^{-3}\right]$ is the surface area concentration of $\mathrm{TiO}_{2}$ particles. 
187 The discrete sectional method is a widely used approach for solving the aerosol general 188 dynamics equation. The particle size distribution is discretized into several discrete sizes in the 189 small size range and is sectionalized in the large size range. In this study, volume concentration 190 is applied as the conserved aerosol moment, which is proven to produce the least numerical 191 diffusion [37]. Based on the TTIP decomposition rate, surface growth rate and the aerosol 192 dynamics equation (1), the DSM scheme proposed by Biswas et. al. [34], with modification to 193 include surface growth, is used to calculate the change in aerosol moment for the discrete sizes 194 and the sections. The number of discrete sizes is set to be 100 , to better reflect the behavior of 195 small $\mathrm{TiO}_{2}$ clusters and to reduce the fluctuation brought by the transition from discrete sizes to 196 sections. Therefore, the discrete sizes cover the volume from $v_{d, 1}\left(=v_{1}\right)$ to $v_{d, 100}\left(=100 v_{1}\right)$, where $197 \mathrm{v}_{1}$ is the volume of a $\mathrm{TiO}_{2}$ monomer. The first section starts from $\mathrm{v}_{\mathrm{s}, 1}\left(=100.5 \mathrm{v}_{1}\right)$ to $\mathrm{v}_{\mathrm{s}, 2}\left(=\mathrm{f}_{\mathrm{s}} \mathrm{v}_{\mathrm{s}, 1}\right)$, 198 where $\mathrm{f}_{\mathrm{s}}$ is the section spacing factor and is set at 1.08 in the simulations. The largest size 199 covered by the sections is $20 \mathrm{~nm}$.

200 Multiple size distributions are obtained at the substrate boundary layer depending on the 201 streamline along which the simulation was performed. As discussed earlier, since the Peclet 202 number is high, diffusion of the aerosols in the gas phase is neglected and hence it is assumed 203 that no interaction takes place between the aerosols followingdifferent streamlines. The boundary 204 layer can be divided into several annuluses based on the discretization of the space by the 205 streamlines and the axisymmetric nature of the reaction chamber (Figure 3b inset). Since, the 206 axial velocity of the flow in every annulus is known, the flow rate through each annulus can be 207 calculated using equation (4):

$$
Q_{i}=v_{i} . A_{i} ; \quad A_{i}=\left(\pi\left(r_{i}+\frac{\Delta r}{2}\right)^{2}-\left(r_{i}-\frac{\Delta r}{2}\right)^{2}\right)
$$


208 where $Q_{i}$ is the flow rate through the $i^{t h}$ annulus with flow velocity $v_{i}$ and a radius $r_{i}$. $\Delta r$ is the

209 difference in radius of two consecutive annuluses. An average arrival particle size distribution

210 was calculated based on the volumetric flow in each annulus represented by the corresponding

211 streamline. The average size distribution is then used for modelling the particle deposition and

212 sintering in the boundary layer.

213 The particles arriving at the boundary layer deposit on the substrate due to Brownian diffusion in

214 the boundary layer. Unlike the flame deposition system, in the ACVD process thermophoresis

215 will reduce the deposition flux.However, for simplicity, the effect of thermophoresis has been

216 ignored since above the boundary layer, the axial flow velocity towards the substrate dominates.

217 Zhang et. al.[33] have shown that the effect of thermophoresis for particles smaller than $2 \mathrm{~nm}$

218 can be ignored in the boundary layer of a flame reactor. The particle flux arriving on the

219 substrate is calculated by solving the 1-D steady state diffusion of particles from the entrance of

220 the boundary layer to the substrate. The particle size distribution obtained from the DSM model

221 and zero number concentration at the substrate are the boundary conditions at the boundary layer

222 entrance and the substrate surface, respectively. The arrival particle fluxobtained is given by:

$$
J\left(d_{p}\right)=\frac{N\left(d_{p}\right) \cdot D\left(d_{p}, T\right)}{L}
$$

223 where $J\left(d_{p}\right)$ is the arrival flux $\left(\# \cdot \mathrm{m}^{-2} \cdot \mathrm{s}^{-1}\right), N\left(d_{p}\right)$ is the number concentration $\left(\# \cdot \mathrm{m}^{-3}\right)$ of the

224 particles with size $d_{p}$ calculated from the discrete sectional simulation, $D\left(d_{p}\right)$ is the diffusivity 225 of the particle $\left(\mathrm{m}^{2} \cdot \mathrm{sec}^{-1}\right)$ reaching the substrate, and $L$ is the thickness of the boundary layer (m).

226 The viscosity of the fluid, the mean free path, and the particle diffusivity are calculated using the 227 average temperature between the substrate and the entrance of the boundary layer.Using the 228 arrival flux, a particle arrival rate in a $200 \mathrm{~nm}$ diameter cross-section (the typical diameter of a 
229 single column in the nanostructured films) is calculated for each particle diameter. As the flux in

230 equation (5) is based on a number concentration that is distributed as per the total discrete sizes

231 and sections, a simple summation yields the total deposition flux. The total arrival flux of

232 particle is calculated according to equation (6):

$$
J_{\text {tot }}=\sum_{i=1}^{100} J\left(d_{p, i}\right)+\sum_{j=1}^{90} J\left(d_{p, j}\right)
$$

233 where $i$ represents the discrete sizes with particle diameter $d_{p, i}$ and jrepresents the sections with 234 midpoint section diameter $d_{p, j}$. A characteristic time for particle arrival $\left(\tau_{a r r}\right)$ is calculated as the 235 inverse of the total particle arrival rate obtained from equation (6).

236 Once particles deposit on the substrate, they sinter with the already deposited particles. Using 237 sintering theory proposed byXing and Rosner [38]and the melting temperature datafor $\mathrm{TiO}_{2}$ 238 reported by Lehtinen et. al [39], a sintering expression similar to one proposed by Tsantilis et. $239 \mathrm{al}[40]$, is derived for $\mathrm{TiO}_{2}$ :

$$
\begin{gathered}
\tau_{\sin }\left(d_{p}\right)=3.01055 \times 10^{15}\left[\frac{\sec }{m \cdot K}\right] d_{p}{ }^{4} T \exp \left(\frac{3.4216 \times 10^{4}}{T}\left(1-\frac{d_{p \min }}{d_{p}}\right)\right) \\
d_{p \min }=\left(\frac{4}{L \cdot \rho_{s}}\right)\left(\sigma_{s}-\sigma_{l}\left(\frac{\rho_{s}}{\rho_{l}}\right)^{\frac{2}{3}}\right)
\end{gathered}
$$

240 where $L$ is the latent heat of fusion per unit volume of solid, $\rho_{s}$ and $\rho_{l}$ arethe solid and liquid phase

241 densities, $\sigma_{s}$ and $\sigma_{l}$ are the solid and liquid surface tensions. The correction factor associated with

242 the particle diameter in the exponential term of equation (8) accounts for the significantly low

243 melting temperatures and viscous flow activation energies for small nanoparticles. As stated by

244 Tsantilis et. al[40], the sintering of particles smaller than $1 \mathrm{~nm}$ (cluster of 15 molecules for $\mathrm{TiO}_{2}$ ) 
245 has been assumed to be instantaneous.Finally, an average sintering time,weighted by the number

246 concentration, is calculated for the particle size distribution arriving at the substrate.

\section{$247 \quad 2.4 \quad$ Experimental validation:}

248 Real time particle size distribution measurements were carried out in the ACVD reactor by a 249 scanning mobility particle sizer comprised of an electrostatic classifier (Model 3082, TSI Inc, 250 MN) fitted with a nano differential mobility analyzer (Nano-DMA, Model 3085, TSI INC, MN), 251 a nano-enhancer (Model 3777, TSI Inc, MN), and a condensation particle counter (Model 3772, 252 TSI Inc, MN) capable of measuring particle size in the range of $1-30 \mathrm{~nm}$. A sampling probe 253 comprised of an 1/8inch tube with a hole drilled in the wall was placed on the substrate hot plate. 254 A sampling flow of $0.4 \mathrm{lpm}$ was taken from the reactor and diluted with filtered air, resulting in a 255 dilution ratio of 20.The size distributions presented are corrected by the dilution ratio used.In 256 order to confirm the model predictions of morphology, the effect of two process parameters, 257 substrate temperature and feed inflow rate, was experimentally analyzed. Substrate temperature 258 in the process was varied in the range of $400^{\circ} \mathrm{C}-600^{\circ} \mathrm{C}$ and the total flow rate was varied in the 259 range 1.0-4.0 $\mathrm{m}^{3} \cdot \mathrm{s}^{-1}$. The morphology of the synthesized thin film was confirmed by scanning 260 electron microscopy (SEM) (FESEM, NOVA NanoSEM 230, FEI Co).

\section{Results and Discussion}

\section{$262 \quad 3.1 \quad$ CFD results for the reaction zone}

263 The velocity and temperature profile obtained from the finite element simulations for the

264 reference case is shown inFigure 3. Simulation results confirm the convective heating of the 265 incoming gas flow as it reaches close to the substrate by the outgoing gas, which exits the reactor 
266 through the spacing between the feeder tube and the quartz glass cover at the top of the reactor.

267 The temperature in the feeder tube and the reaction chamber is fairly uniform and increases

268 rapidly near the substrate.

269 The velocity streamlines in the reactor are shown inFigure 3c. The streamlines highlight the 270 complex geometry of the ACVD reactor flow impinging on the substrate, turning radially and

271 then back again due to the geometry of the outer quartz glass chamber. This causes a 272 recirculation region in the reactor where prediction of aerosol dynamics is further 273 complicated.Above the substrate, the velocitystreamlines turn radially creating a stagnation 274 region where diffusion is the dominant mechanism for particle transport to the substrate.

275 Figure 3dshows the velocity and temperature profile at the axisymmetric axis (center of the 276 substrate). The boundary layer height is defined to be $0.06 \mathrm{~mm}$ above the substrate,where it is 277 seen that the axial velocity is less than $1 \%$ of the velocity magnitude. The streamlines entering 278 the boundary later are shown in Figure 3c (right), along which the discrete sectional simulations 279 are carried out. It is further observed that the temperature rises rapidly in the $0.7 \mathrm{~mm}$ region 280 above the substrate, which indicates that the reaction and aerosol dynamics in this region play a 281 major role in governing depositing particle size.

\section{$282 \quad 3.2$ Simulation results for data in literature:}

283 The process of particle diffusion, deposition, and sintering to yield a nanostructured thin film 284 takes place in the substrate boundary layer. These processes depend on the particle size arriving 285 at the boundary layer, the number concentration of the particles, and the temperature of the 286 substrate. The simulated size distributions of particles arriving at the substrate boundary layer, 287 obtained using the three sets of process parameters (Test 1, 4, 8) reported by An et al.[1], are 
shown in Figure 4a. Each of the three cases represents either a dense, columnar or granular

289 morphology, as defined by the previous study. It is observed that the morphology is correlated to

290 the size distribution, with a shift towards the larger size corresponding to a transition of 291 morphology from dense to columnar to granular. Further, it was observed that the particle flux 292 arriving at the boundary layer increases significantly from $1.28 \times 10^{9} \# / \mathrm{s}$ for the dense film to $2934.50 \times 10^{10} \# / s$ for the columnar film and $9.07 \times 10^{10} \# / s$ for the granular film. Thus, the larger size 294 distribution, along with a higher particle flux arriving at the boundary layer, leads to a significant 295 change in morphology. The model predicted results were confirmed by the experimental 296 measurement of particle size distribution in the ACVD reactor (Figure 4b). Although the 297 experimental measurements portray a larger particle size, possibly due to particle coagulation 298 and growth in the sampling tube, the trends in number concentration are similar to the model 299 predicted results. Overall, a significant increase in the total number concentration was observed 300 in the columnar and granular case as compared to the dense case.

301 While surface reaction of the vapor on the substrate is the dominant growth mechanism, the 302 morphology is governed by the presence of particles in the depositing mix. In order to 303 quantitatively correlate the particle size distribution and flux arriving at the boundary layer to the 304 film morphology, a characteristic time ratio (CTR) is defined as the ratio of the characteristic 305 time for particle arrival $\left(\tau_{a r r}\right)$ to the characteristic time for particle sintering $\left(\tau_{\sin }\right)$. The $\tau_{\sin }$ is an 306 average sintering time for the different particle sizes weighted by the number concentration of 307 the corresponding particle size. The CTR takes into account all factors governing the 308 morphology of the thin film including, the particle size distribution, the particle arrival flux, and 309 the sintering rate of the particles. A similar approach has been used previously by Lehtinen et al. 310 to describe the onset of dendrite formation in $\mathrm{TiO}_{2}$ particlesas a result of coalescence and 
311 sintering in the gas phase [39]. The values for the dense, columnar, and granular film was

312 calculated to be $6.1 \times 10^{25}, 4.8 \times 10^{1}$, and $1.06 \times 10^{-2}$ respectively. This agrees with the previous

313 understanding that the dense morphology is a result of predominantly vapor deposition,

314 extremely long particle deposition time and ultrafast sintering times; the columnar morphology is

315 a result of comparable arrival times and sintering times leading to the arrival of the particle and

316 near complete sintering with the existing structure allowing for the formation of a single crystal

317 structure; and the granular morphology is a result of highly incomplete sintering leading to

318 stacking of particles with identifiable grain boundaries[1].

319 3.3 The influence of process parameters on particle size distribution and $320 \quad$ morphology

321 As described earlier, the process of nanostructure thin film synthesis in the ACVD system can be 322 divided into two stages. The first stage is particle formation and growth in the gas phase, 323 followed by the second stage where particle deposition and film formation take place in the 324 substrate boundary layer. The size distribution of the particles arriving at the substrate boundary 325 layer, the final result of particle evolution in the gas phase and the initial condition for film 326 formation in the latter stage, plays a connecting role in the relationship between the process 327 parameters and the final film morphology. In order to achieve a controlled morphology, an 328 understanding of the influence of process parameters on the arrival particle size distribution and 329 the CTR is needed.

330 The arrival particle size distribution obtained from the coupled finite element - discrete sectional 331 simulation is a result of the particle evolution processthat is dictated by the complex combination 332 of various aerosol dynamics occurring in the reaction chamber. In order to assess and explain the 
333 influence of the ACVD process parameters on the final particle size, two governing factors are

334 extracted from the CFD simulation results: the time averaged temperature $\left(T_{\text {avg }}\right)$ and the

335 residence time $\left(t_{r e s}\right)$ of the aerosol. The residence time indicates the time for which aerosol 336 dynamics have been ongoing in the reactor and thus determines the final particle size. In the 337 present case, particle formation and growth predominantly takes place after the precursor gas 338 exits the feeder tube, which was earlier defined as the refined zone in Figure 1b. Hence the 339 residence time has been defined as the time it takes for the precursor gas to flow from the tube 340 exit to the boundary layer surface $\left(t_{r e s}=\sum t_{i}\right)$. The time averaged temperature can be used to 341 evaluate the temperature history of the aerosol, which will determine the rate of aerosol 342 dynamics. Due to the temporal nature of the aerosol dynamics, the temperature has been 343 averaged on the time scale $\left(T_{\text {avg }}=\sum t_{i} T_{i} / t_{\text {res }}\right)$. In the present case, similar to the residence 344 time, the time averaged temperature history has been calculated for the refined zone. These two 345 governing factors are used to explain the influence of ACVD process parameters on the particle 346 size distribution arriving at the boundary layer.

347 Figure 5shows the influence of substrate temperature. With an increase in the substrate 348 temperature, the arrival particle size distribution shifts towards larger sizes and a higher number 349 concentration. This trend is attributed to the rise of the time averaged particle temperature as the 350 substrate temperature rises (Figure 5b). The residence time also appears to decrease slightly with 351 an increase in substrate temperature. Trends of these two factors have opposing effects on the 352 particle size distribution; however, the change in time averaged temperature dominates, leading 353 to the aforementioned trend in the particle size distribution. In addition, we also note that the 354 change in size distribution is more rapid as the substrate temperature rises. It reflects that the 355 time averaged temperature has a nonlinear effect on the particle size distribution, which likely 
356 originates from the exponential dependence of the rate of precursor decomposition on the

357 temperature. The change in particle size distribution affects both the $\tau_{a r r}$ and $\tau_{\text {sin }}$ since both

358 these characteristic times are a function of the particle size. The change in substrate temperature

359 also influences the $\tau_{\text {sin }}$ directly because sintering rate depends on the temperature of the

360 substrate in addition to the particle size as shown in eq. (7). In addition, a continuous decrease in

361 the CTR is observed with an increase in temperature (Figure 5c), which can be attributed to the

362 increasing particle flux (decreasing $\tau_{a r r}$ ) accompanied by an increasing $\tau_{\sin }$ seen for larger

363 particles. The effect of substrate temperature was experimentally verified, as shown inFigure

364 5d,by synthesizing nanostructured thin films keeping all of the process parameters for columnar

365 conditions butvarying the substrate temperatures from $400^{\circ} \mathrm{C}-600^{\circ} \mathrm{C}$. In all cases, a columnar

366 morphology was observed by SEM, but the gradual transition from granular to columnar may not

367 be visible throughthis characterization technique. Model predictions at $600^{\circ} \mathrm{C}$ are closer to the

368 granular CTR while the morphology observed experimentally is columnar. This deviation is

369 likely due to the effect of thermophoresis at higher temperatures on the depositing particle size

370 distribution which have not been accounted for in the present work. Thermophoresis will reduce

371 the deposition flux and at higher temperatures, due to higher temperature gradients, the effect is

372 more pronounced. Branching of a few tall nanostructures was observed for the $400^{\circ} \mathrm{C}$ substrate

373 temperature. This phenomenon of branching has been previously studied in detail and is a

374 function of the thermal conductivity of the $\mathrm{TiO}_{2}$ columns, which results in lower temperature at

375 the tip of the column and hence lowers the sintering rate,resulting in the formation of 376 branches[41].

377 The results of the effect of feeder-substrate distance are shown in Figure 6, which indicates that 378 the arrival particle size distribution is only slightly affected by the feeder substrate distance. The 
379 lack of any significant effect of this parameter occurs due to the strong opposition between the

380 increase in time averaged particle temperature and the corresponding decrease in the residence

381 time (Figure 6b). This also corresponds to a decrease in the $\tau_{\text {arr }} / \tau_{\sin }$ (Figure 6c), however this

382 decrease is not thought to be significant enough to cause an observable change in the 383 morphology.

384 The other three process parameters, $\mathrm{Q}_{0}, \mathrm{~T}_{0}$ and $\mathrm{c}_{0}$, are properties of the precursor flow, which is 385 controlled by the precursor feed system. Figure 6a shows the influence of total flow rate on the 386 arrival particle size distribution. In all cases, the precursor concentration was maintained constant 387 at the reference value. Increase in the flow rateresults in a decrease in the arrival particle size and 388 the number concentration resulting in a left shift in the arrival particle size distribution (Figure 389 7a). This shift is a result of the decrease in both the time averaged temperature and residence 390 time (Figure 7b). These two factors decelerate precursor decomposition and particle growth, and 391 therefore the particle size and concentration drops sharply. This sharp drop is also reflected in a 392 rapid decrease in the CTR, which allows the morphology to be changed just by changing the feed 393 flow rate (Figure 7c). The effect of the total flow rate on the morphology of the synthesized 394 nanostructure was also verified experimentally. Decreasing the total flow rate to $1.0 \mathrm{~m}^{3} . \mathrm{s}^{-1}$ causes 395 the morphology to change from columnar to completely granular, which is in agreement with the 396 simulation. Increasing the feed flow rate to $4.0 \mathrm{~m}^{3} . \mathrm{s}^{-1}$ causes the morphology to change to an 397 irregular dense morphology, again in agreement with the simulation prediction.

398 Figure 8a presents the influence of initial precursor concentration on the arrival particle size 399 distribution. Due to the low precursor concentration in the feed flow, the concentration of the 400 precursor does not change the fluid flow and energy balance, and hence the time averaged 401 temperature and residence time do not change with the initial precursor concentration. However, 
402 the initial precursor concentration indeed has influence on the particle size distribution, which 403 implies that initial precursor concentration also serves as an essential factor that governs particle 404 evolution. Since the precursor decomposition reaction rate is first order with respect to the 405 precursor, higher precursor concentration leads to a higher generation of $\mathrm{TiO}_{2}$ monomers, and 406 hence results in a higher number concentration and larger particle size, as shown by the 407 simulation results. This effect is translated to the decrease in the CTR with increasing precursor 408 concentration, which can be used to control the morphology of the thin film (Figure 8b).

409 Figure 9a shows the influence of feed inlet temperature on the arrival particle size distribution.

410 The increase of feed inlet temperature leads to an increase of both the time averaged temperature 411 and residence time (due to change in gas density) (Figure 9b). However, the change in time 412 averaged temperature is again dominant in comparison to the residence time, so the influence of 413 feed temperature is similar with that of the substrate temperature. However, the effect of the feed 414 inlet temperature on the CTR is slightly different than the effect of substrate temperature. Since 415 substrate temperature also influence surface sintering rates, the slope of the decrease in CTR 416 changed at higher temperatures while the decrease in CTR for the precursor inlet temperature is 417 more uniform. (Figure 9c).

418 In summary, the time averaged particle temperature, residence time and the initial precursor 419 concentration are three essential factors that govern particle evolution, which could be used to 420 explain and predict the influence of process parameters. Among the five process parameters, the 421 increase of substrate temperature, initial precursor concentration, and feeding flow temperature 422 promotes aerosol generation and growth,resulting in an increase in the arrival particle size and 423 the total number concentration. Alternatively, increasingthe feed flow rate has the opposite 424 effect. All process parameters influence the morphology consistent with their influence on the 
425 arrival particle size distribution. Interestingly, the feeder substrate distance has no significant

426 effect on the arrival particle size distribution and hence the morphology of the thin film.

\section{Conclusions:}

428 A finite element based computational fluid dynamics model was used to simulate the 429 temperature and velocity profile in the ACVD reaction, which was then coupled to a discrete 430 sectional model to obtain the particle size distribution evolution. A characteristic time ratio

431 (CTR), $\tau_{a r r} / \tau_{\sin }$ was then calculated to predict the morphology of the nanostructured thin film.

432 The effect of process parameters on the morphology was predicted and an understanding of these 433 effects was developed based on the particle size distribution, the time averaged temperature, and 434 the residence time in the reactor. The prediction of morphology obtained by the model was 435 experimentally verified for two process parameters. The model provides key insights into the 436 role of fluid and aerosol dynamics, and the ability to predict nanostructure morphology in the 437 ACVD process. This could be used to provide a better control over the morphology of the 438 synthesized films and help devise strategies to improve the throughput of the process for scaled439 up production. Further, this modelling approach can be extended to other gas phases deposition 440 processes such as the flame aerosol deposition process and the electrospray deposition process.

\section{Acknowledgments:}

442 This paper is based upon work supported by the Solar Energy Research Institute for India and the 443 U.S. (SERIIUS) funded jointly by the U.S. Department of Energy subcontract DE AC36444 08G028308 (Office of Science, Office of Basic Energy Sciences, and Energy Efficiency and 445 Renewable Energy, Solar Energy Technology Program, with support from the Office of 446 International Affairs) and the Government of India subcontract IUSSTF/JCERDC- 
SERIIUS/2012 dated 22nd Nov. 2012. Electron microscopy was performed at the Nano Research

448 Facility (NRF) at Washington University in St. Louis, a member of the National Nanotechnology

449 Infrastructure Network (NNIN), supported by the National Science Foundation under Grant No.

450 ECS-0335765. The work of MMY and SQL was supported by the National Natural Science

451 Funds of China (Nos. 51390491 and51428602), and by the National Key Basic Research and

452 Development Program (2013CB228506).

\section{References}

454 [1] W.-J. An, E. Thimsen, P. Biswas, Aerosol-chemical vapor deposition method for synthesis of 455 nanostructured metal oxide thin films with controlled morphology, J. Phys. Chem. Lett. 1 (2009) $456 \quad 249-253$.

457 [2] E. Thimsen, N. Rastgar, P. Biswas, Nanostructured TiO2 films with controlled morphology 458 synthesized in a single step process: Performance of dye-sensitized solar cells and photo 459 watersplitting, J. Phys. Chem. C 112 (2008) 4134-4140.

460 [3] A. Listorti, E.J. Juarez-Perez, C. Frontera, V. Roiati, L. Garcia-Andrade, S. Colella, A. Rizzo, 461 P. Ortiz, I. Mora-Sero, Effect of mesostructured layer upon crystalline properties and device 462 performance on perovskite solar cells, J. Phys. Chem. Lett. 6 (2015) 1628-1637.

463 [4] L.B. Modesto-Lopez, E.J. Thimsen, A.M. Collins, R.E. Blankenship, P. Biswas, 464 Electrospray-assisted characterization and deposition of chlorosomes to fabricate a biomimetic 465 light-harvesting device, Energy Environ. Sci. 3 (2010) 216-222.

466 [5] A. Kay, I. Cesar, M. Grätzel, New benchmark for water photooxidation by nanostructured $\alpha$ 467 Fe2O3 films, J. Am. Chem. Soc. 128 (2006) 15714-15721.

468 [6] T.S. Chadha, J. Park, W.J. An, P. Biswas, Gold nanocage coupled single crystal TiO2 469 nanostructures for near-infrared water photolysis, J. Nanopart. Res. 16 (2014) 1-9. 
470 [7] G.K. Mor, O.K. Varghese, R.H. Wilke, S. Sharma, K. Shankar, T.J. Latempa, K.-S. Choi, 471 C.A. Grimes, p-Type $\mathrm{Cu}^{-} \mathrm{Ti}-\mathrm{O}$ nanotube arrays and their use in self-biased heterojunction 472 photoelectrochemical diodes for hydrogen generation, Nano Lett. 8 (2008) 1906-1911.

473 [8] R. van de Krol, Y. Liang, J. Schoonman, Solar hydrogen production with nanostructured 474 metal oxides, J. Mater. Chem. 18 (2008) 2311-2320.

475 [9] I. Cesar, K. Sivula, A. Kay, R. Zboril, M. Grätzel, Influence of feature size, film thickness, 476 and silicon doping on the performance of nanostructured hematite photoanodes for solar water 477 splitting, J. Phys. Chem. C 113 (2008) 772-782.

478 [10] S. Kavadiya, T.S. Chadha, H. Liu, V.B. Shah, R.E. Blankenship, P. Biswas, Directed 479 assembly of the thylakoid membrane on nanostructured TiO 2 for a photo-electrochemical cell, $480 \quad$ Nanoscale 8 (2016) 1868-1872.

481 [11] V.B. Shah, W.R. Henson, T.S. Chadha, G. Lakin, H. Liu, R.E. Blankenship, P. Biswas, 482 Linker-Free Deposition and Adhesion of Photosystem I onto Nanostructured TiO2 for Biohybrid 483 Photoelectrochemical Cells, Langmuir 31 (2015) 1675-1682.

484 [12] L. Liu, D.T. Pitts, H. Zhao, C. Zhao, Y. Li, Silver-incorporated bicrystalline 485 (anatase/brookite) TiO 2 microspheres for $\mathrm{CO} 2$ photoreduction with water in the presence of 486 methanol, Appl. Catal., A 467 (2013) 474-482.

487 [13] W.-N. Wang, W.-J. An, B. Ramalingam, S. Mukherjee, D.M. Niedzwiedzki, S. 488 Gangopadhyay, P. Biswas, Size and structure matter: enhanced CO2 photoreduction efficiency 489 by size-resolved ultrafine Pt nanoparticles on TiO2 single crystals, J. Am. Chem. Soc. 134 $490 \quad$ (2012) 11276-11281. 
491 [14] T.S. Chadha, A.M. Tripathi, S. Mitra, P. Biswas, One

492 single abryiø2 nanostructured anodes synthesized by a single -dimensional,

-step aerosol

493 high

-9atte lithium - ion batteries, Energy Technol. 2 (

494 [15] S. Banerjee, Y. Myung, S. Raman, P. Banerjee, Direct growth of flexible and scalable 495 photocathodes from $\alpha$-brass substrates, ACS Sustainable Chem. Eng. 3 (2015) 3197-3204.

496 [16] Y. Wu, P. Yang, Direct observation of vapor-liquid-solid nanowire growth, J. Am. Chem. 497 Soc. $123(2001)$ 3165-3166.

498 [17] A. Huczko, Template-based synthesis of nanomaterials, Appl. Phys. A 70 (2000) 365-376.

499 [18] S. Banerjee, Y. Myung, P. Banerjee, Confined anodic aluminum oxide nanopores on 500 aluminum wires, RSC Adv. 4 (2014) 7919-7926.

501 [19] E. Thimsen, P. Biswas, Nanostructured photoactive films synthesized by a flame aerosol 502 reactor, AIChE J. 53 (2007) 1727-1735.

503 [20] M. Kubo, Y. Ishihara, Y. Mantani, M. Shimada, Evaluation of the factors that influence the 504 fabrication of porous thin films by deposition of aerosol nanoparticles, Chem. Eng. J. 232 (2013) $505 \quad 221-227$.

506 [21] H. Komiyama, T. Osawa, Rapid Growth of AlN Films by Particle-Precipitation Aided 507 Chemical Vapor Deposition, Jpn. J. Appl. Phys. 24 (1985) L795.

508 [22] T.S. Chadha, P. Biswas, W.-j. An, Single-step synthesis of nanostructured thin films by a 509 chemical vapor and aerosol deposition process, US Patent 20,160,056,448, 2016.

510 [23] K. Haddad, A. Abokifa, S. Kavadiya, T.S. Chadha, P. Shetty, Y. Wang, J. Fortner, P. 511 Biswas, Growth of single crystal, oriented $\mathrm{SnO} 2$ nanocolumn arrays by aerosol chemical vapour 512 deposition, CrystEngComm 18 (2016) 7544-7553. 
513 [24] A. Schild, A. Gutsch, H. Mühlenweg, S. Pratsinis, Simulation of nanoparticle production in

514 premixed aerosol flow reactors by interfacing fluid mechanics and particle dynamics, J.

515 Nanopart. Res. 1 (1999) 305-315.

516 [25] A.J. Gröhn, S.E. Pratsinis, K. Wegner, Fluid-particle dynamics during combustion spray

517 aerosol synthesis of ZrO 2, Chem. Eng. J. 191 (2012) 491-502.

518 [26] C.-Y. Wu, P. Biswas, Lead species aerosol formation and growth in multicomponent high-

519 temperature environments, Environ. Eng. Sci. 17 (2000) 41-60.

520 [27] E.R. Whitby, P.H. McMurry, Modal aerosol dynamics modeling, Aerosol Sci. Technol. 27

521 (1997) 673-688.

522 [28] D.R. Warren, J.H. Seinfeld, Simulation of aerosol size distribution evolution in systems

523 with simultaneous nucleation, condensation, and coagulation, Aerosol Sci. Technol. 4 (1985) 31-

52443.

525 [29] J.J. Wu, R.C. Flagan, A discrete-sectional solution to the aerosol dynamic equation, J.

526 Colloid Interface Sci. 123 (1988) 339-352.

527 [30] Z. Sun, R. Axelbaum, B. Chao, A multicomponent sectional model applied to flame 528 synthesis of nanoparticles, Proc. Combust. Inst. 29 (2002) 1063-1069.

529 [31] A.S. Wexler, F.W. Lurmann, J.H. Seinfeld, Modelling urban and regional aerosols-I.

530 Model development, Atmos. Environ. 28 (1994) 531-546.

531 [32] A.J. Gröhn, S.E. Pratsinis, K. Wegner, Fluid-particle dynamics during combustion spray 532 aerosol synthesis of ZrO2, Chem. Eng. J. 191 (2012) 491-502.

533 [33] Y. Zhang, S. Li, W. Yan, Q. Yao, Nanoparticle transport and deposition in boundary layer 534 of stagnation-point premixed flames, Powder Technol. 227 (2012) 24-34. 
535 [34] P. Biswas, C.Y. Wu, M.R. Zachariah, B. McMillin, Characterization of iron oxide-silica

536 nanocomposites in flames: Part II. Comparison of discrete-sectional model predictions to 537 experimental data, J. Mater. Res. 12 (1997) 714-723.

538 [35] K. Nakaso, K. Okuyama, M. Shimada, S.E. Pratsinis, Effect of reaction temperature on 539 CVD-made TiO 2 primary particle diameter, Chem. Eng. Sci. 58 (2003) 3327-3335.

540 [36] S. Tsantilis, H.K. Kammler, S.E. Pratsinis, Population balance modeling of flame synthesis 541 of titania nanoparticles, Chem. Eng. Sci. 57 (2002) 2139-2156.

542 [37] C.-Y. Wu, P. Biswas, Study of numerical diffusion in a discrete-sectional model and its 543 application to aerosol dynamics simulation, Aerosol Sci. Technol. 29 (1998) 359-378.

544 [38] Y. Xing, D.E. Rosner, Prediction of spherule size in gas phase nanoparticle synthesis, J. 545 Nanopart. Res. 1 (1999) 277-291.

546 [39] K.E. Lehtinen, R.S. Windeler, S.K. Friedlander, Prediction of nanoparticle size and the 547 onset of dendrite formation using the method of characteristic times, J. Aerosol Sci. 27 (1996) $548 \quad 883-896$.

549 [40] S. Tsantilis, H. Briesen, S. Pratsinis, Sintering time for silica particle growth, Aerosol Sci. 550 Technol. 34 (2001) 237-246.

551 [41] W.-J. An, D.D. Jiang, J.R. Matthews, N.F. Borrelli, P. Biswas, Thermal conduction effects 552 impacting morphology during synthesis of columnar nanostructured TiO2 thin films, J. Mater. 553 Chem. 21 (2011) 7913-7921. 
Table 1: List of process parameters and their values used in the simulation study

\begin{tabular}{|c|c|c|}
\hline Process Parameter & Simulation Range & Reference Value \\
\hline Substrate Temperature $\mathbf{( \mathbf { T } _ { \mathbf { s } } )}$ & $300-700^{\circ} \mathrm{C}$ & $450^{\circ} \mathrm{C}$ \\
\hline Feeder Substrate Distance $\left(\mathbf{h}_{\mathbf{0}}\right)$ & $5-40 \mathrm{~mm}$ & $20 \mathrm{~mm}$ \\
\hline Feed Flow Rate $\left(\mathbf{Q}_{\mathbf{0}}\right)$ & $0.5 \times 10^{-5}-2.5 \times 10^{-5} \mathrm{~m}^{3} \cdot \mathrm{s}^{-1}$ & $1.83 \times 10^{-5} \mathrm{~m}^{3} \cdot \mathrm{s}^{-1}$ \\
\hline Precursor Concentration $\left(\mathbf{c}_{\mathbf{0}}\right)$ & $1 \times 10^{-3}-1 \times 10^{-2} \mathrm{~mol}^{-\mathrm{m}^{-3}}$ & $1.51 \times 10^{-3} \mathrm{~mol}^{-3}$ \\
\hline Feed Inlet Temperature $\left(\mathbf{T}_{\mathbf{0}}\right)$ & $20-100^{\circ} \mathrm{C}$ & $35^{\circ} \mathrm{C}$ \\
\hline
\end{tabular}




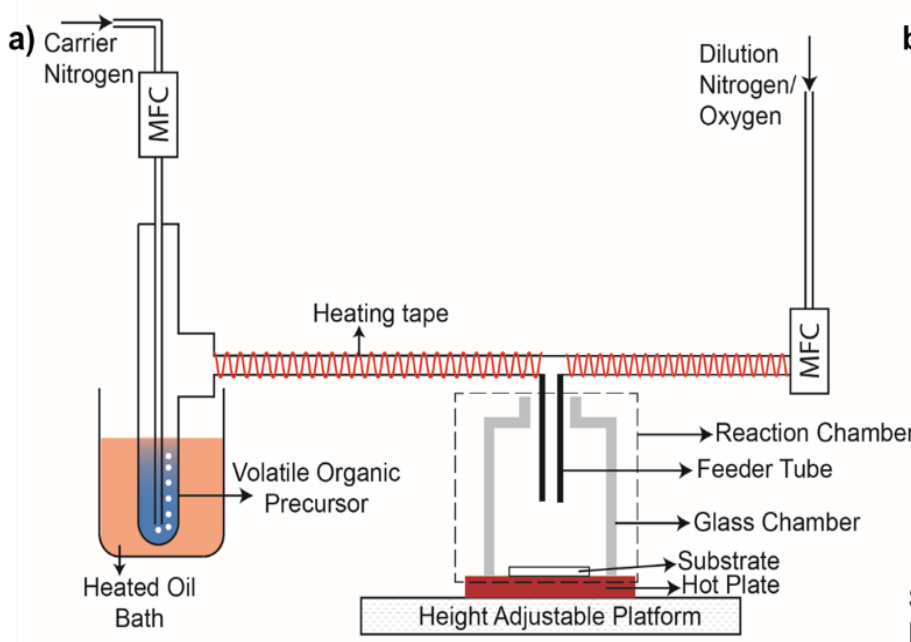

Figure 1: Schematic of the (a) ACVD process and (b) 2D axisymmetric setup of the ACVD reactor for the finite element modelling 


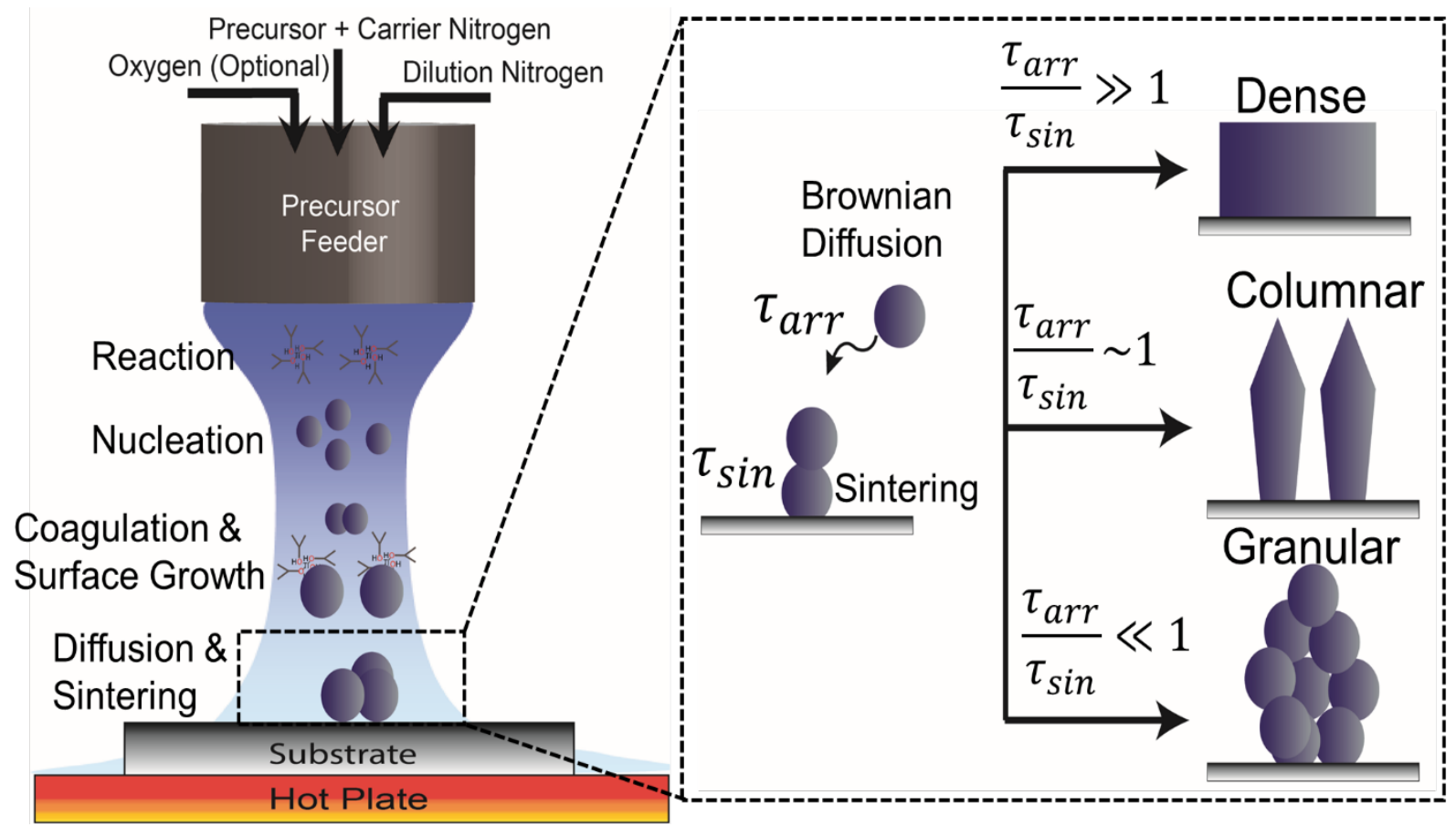

Figure 2: Overview of aerosol dynamics simulated by the discrete sectional model, the Brownian dynamics model and the sintering model to predict film morphology synthesized by the ACVD process 
a)

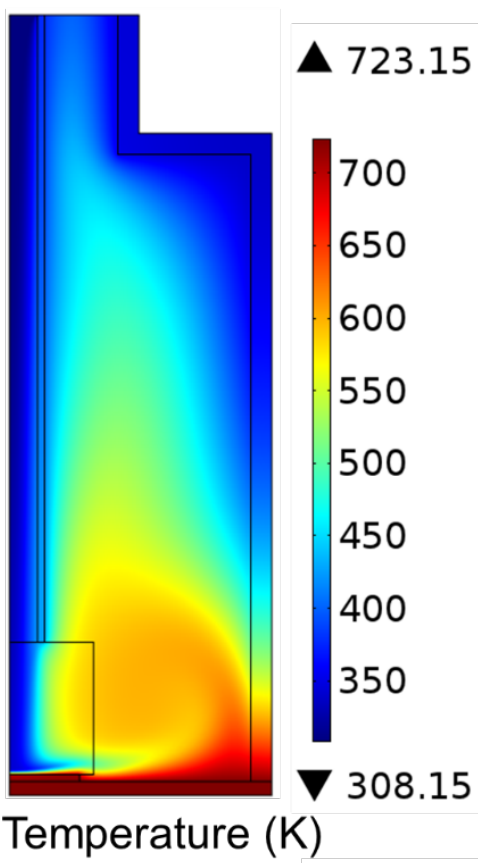

b)

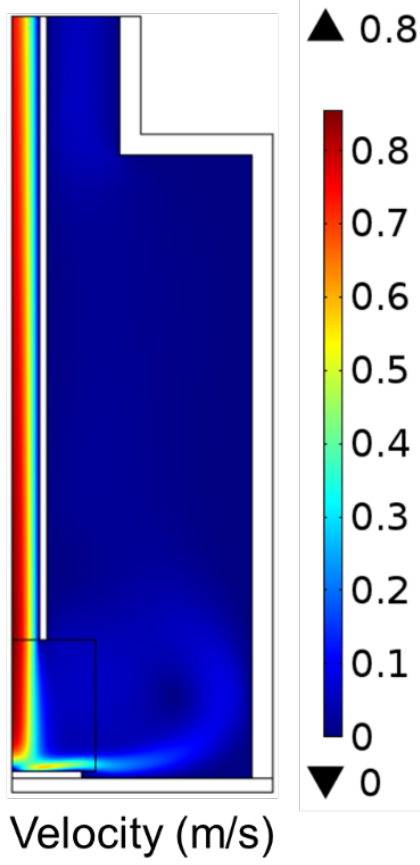

c)

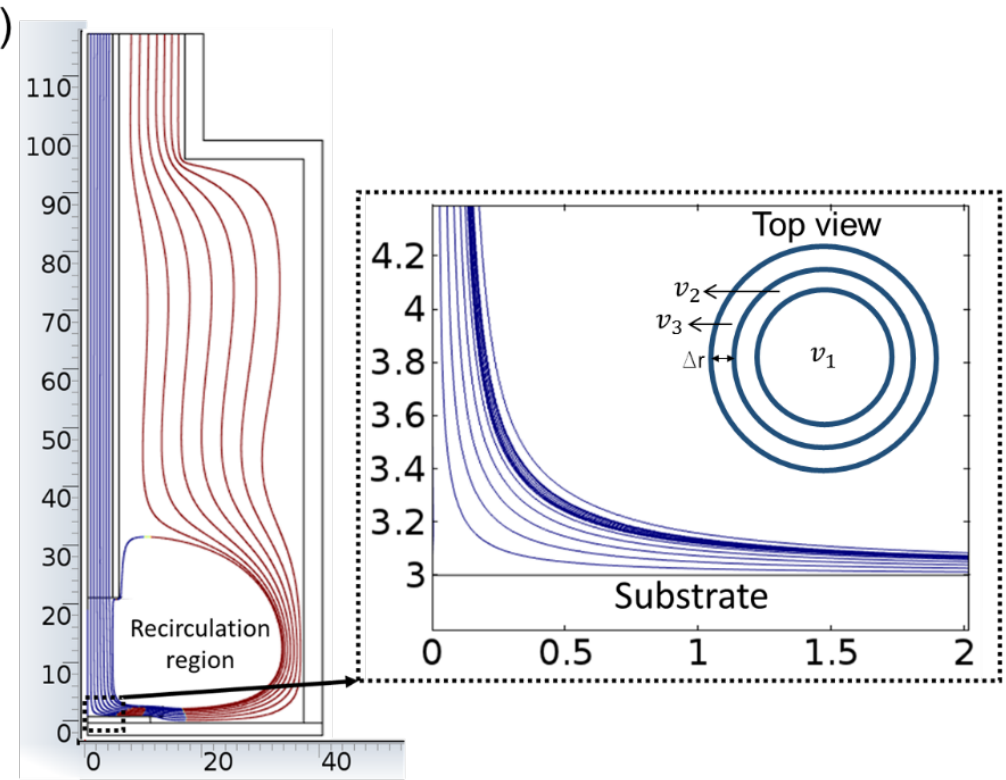

d)

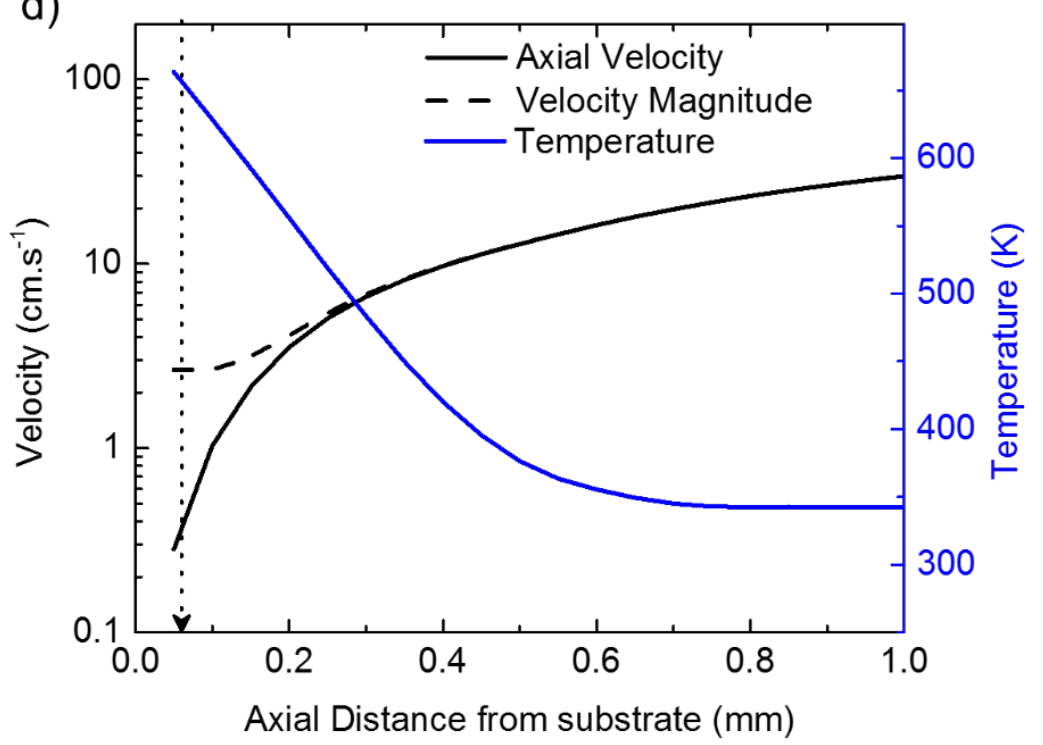

Figure 3: (a) Temperature,(b) velocity profile,(c) velocity streamlines depicting flow pattern in the ACVD reactor, and(d) velocity and temperature profile above the center of the substrate obtained from the finite element simulation 


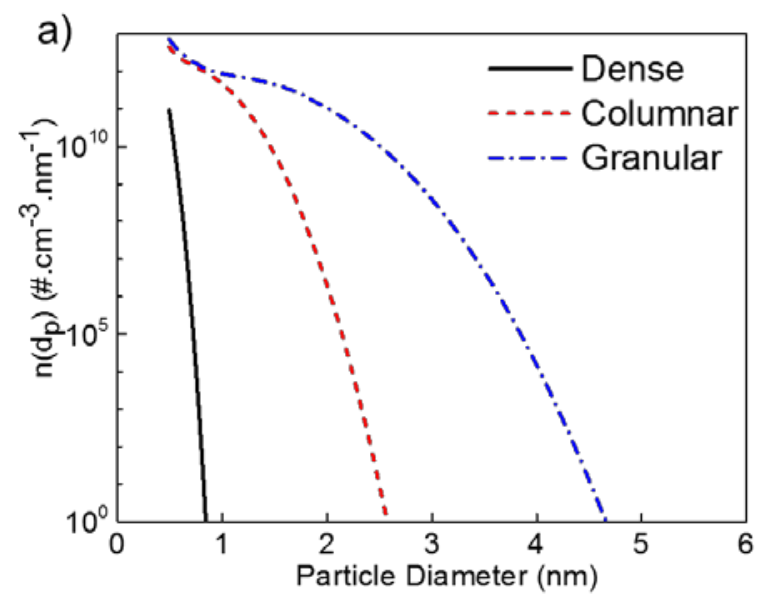

b)

\begin{tabular}{c|c|c}
\hline Morphology & $\begin{array}{c}\text { Geometric } \\
\text { Mean Diameter } \\
(\mathbf{n m})\end{array}$ & $\begin{array}{c}\text { Total number } \\
\text { concentration } \\
\left(\# . \mathbf{c m}^{-3}\right)\end{array}$ \\
\hline Dense & 5.93 & $6.95 \times 10^{7}$ \\
\hline Columnar & 5.96 & $7.98 \times 10^{8}$ \\
\hline Granular & 7.02 & $7.97 \times 10^{8}$ \\
\hline
\end{tabular}

Figure 4: (a) Simulated and (b) experimentally measured particle size distribution corresponding to the three different morphologies. Results reported are obtained from simulation and experiments performed at operating conditions describedby An et. al[1] 

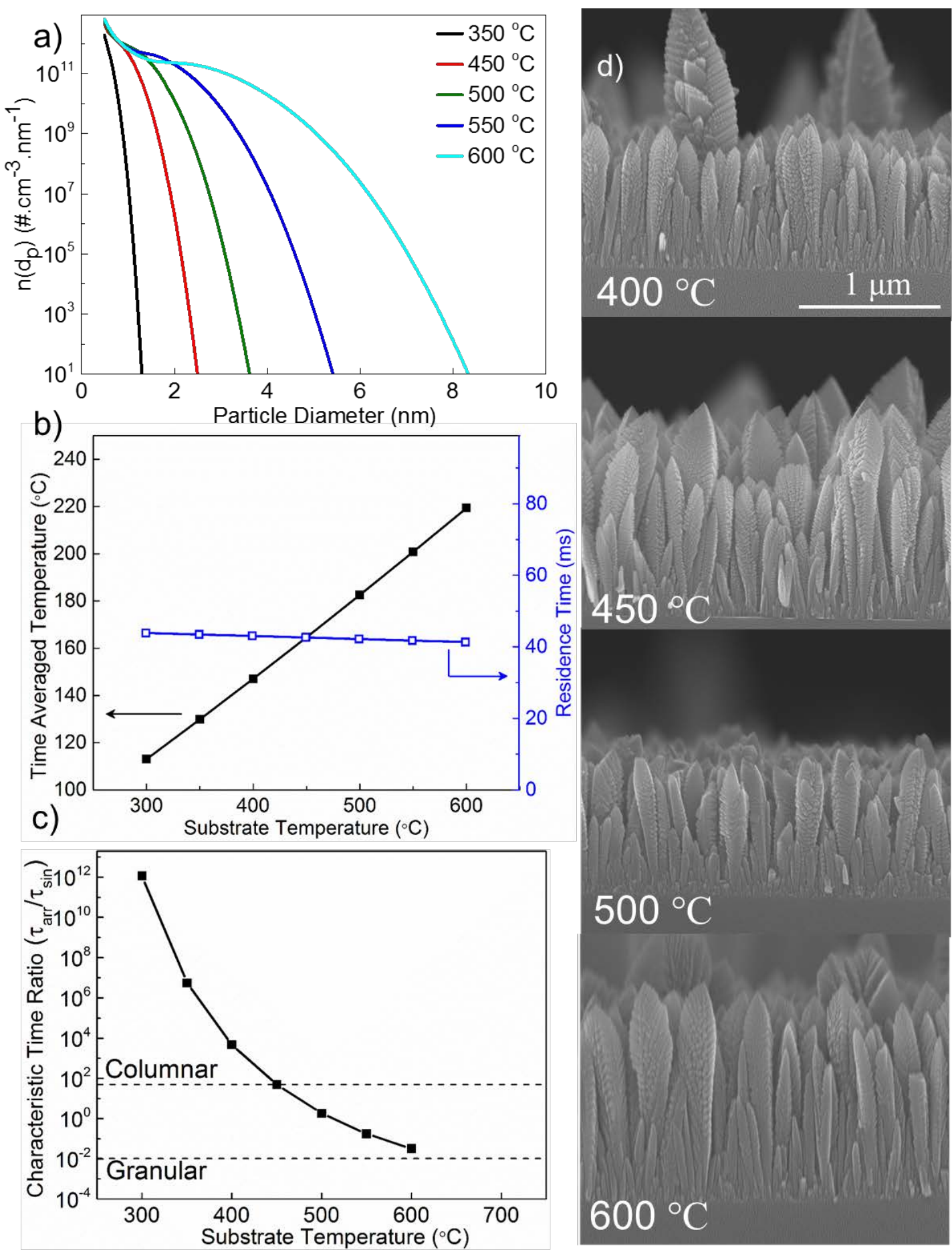

Figure 5: Effect of substrate temperature on (a) the particle size distribution (b) the time averaged temperature and (c) characteristic time ratio. The dotted lines indicate the value of the characteristic time ratio $\left(\tau_{\text {arr }} / \tau_{\sin }\right)$ for different morphologies observed experimentally by An et. al. [1] (d) SEM images depicting the morphology of the thin films synthesized at various temperatures 

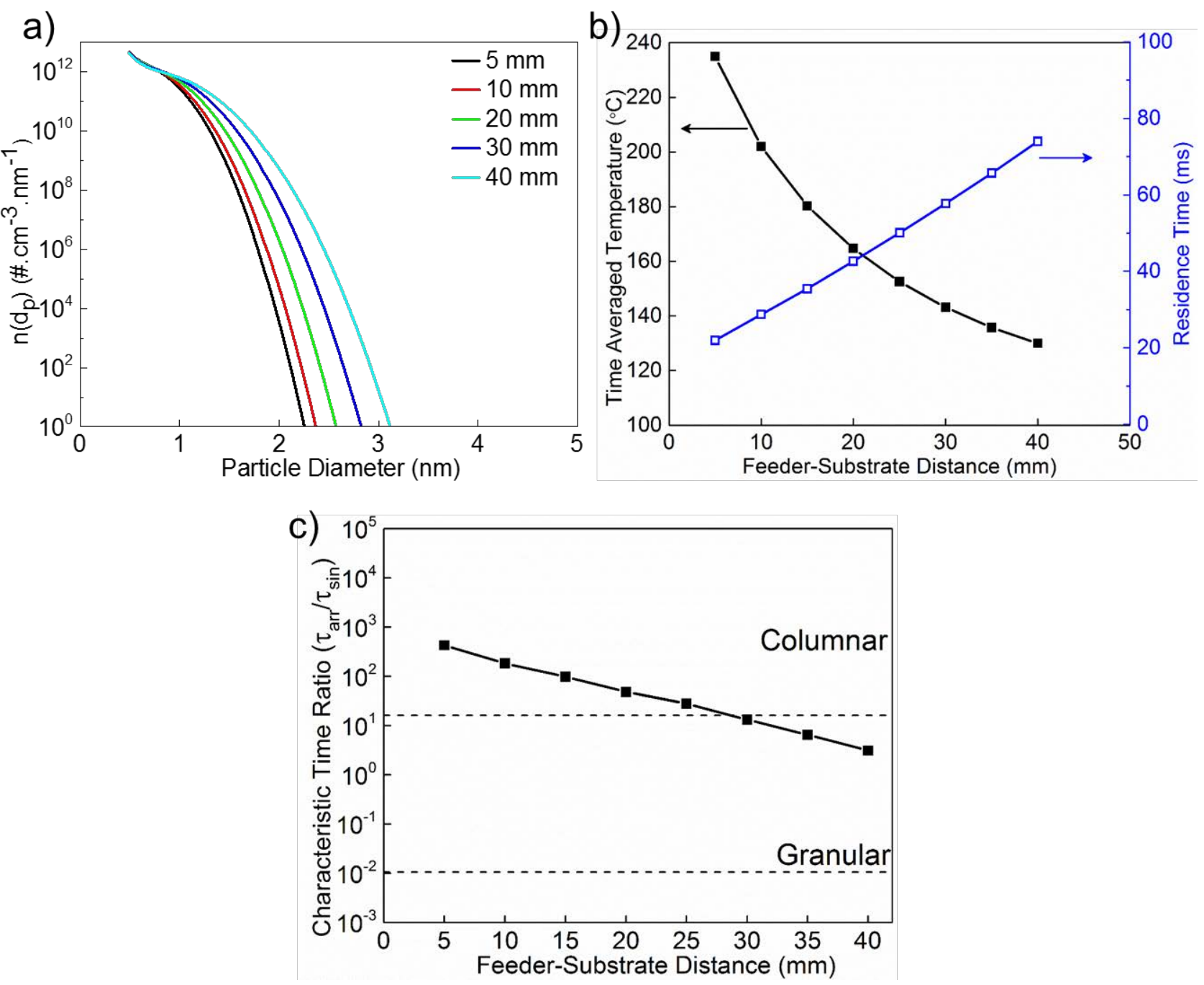

Figure 6: Effect of feeder-substrate distance on (a) the particle size distribution (b) the time averaged temperature and (c) characteristic time ratio 
a)

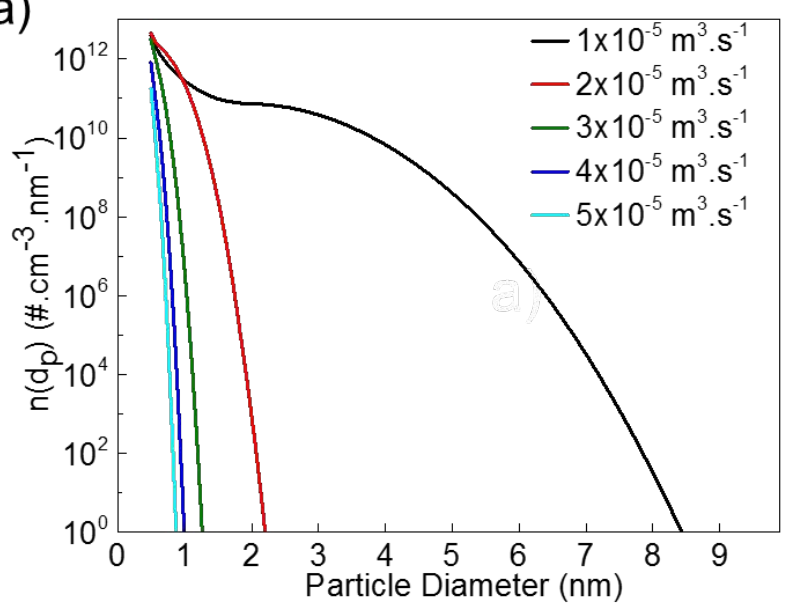

b)

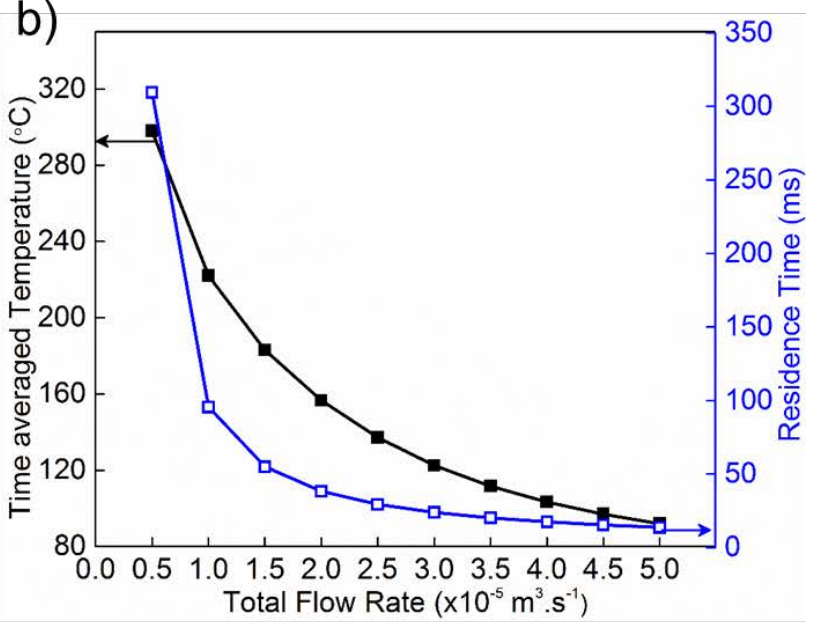

c)

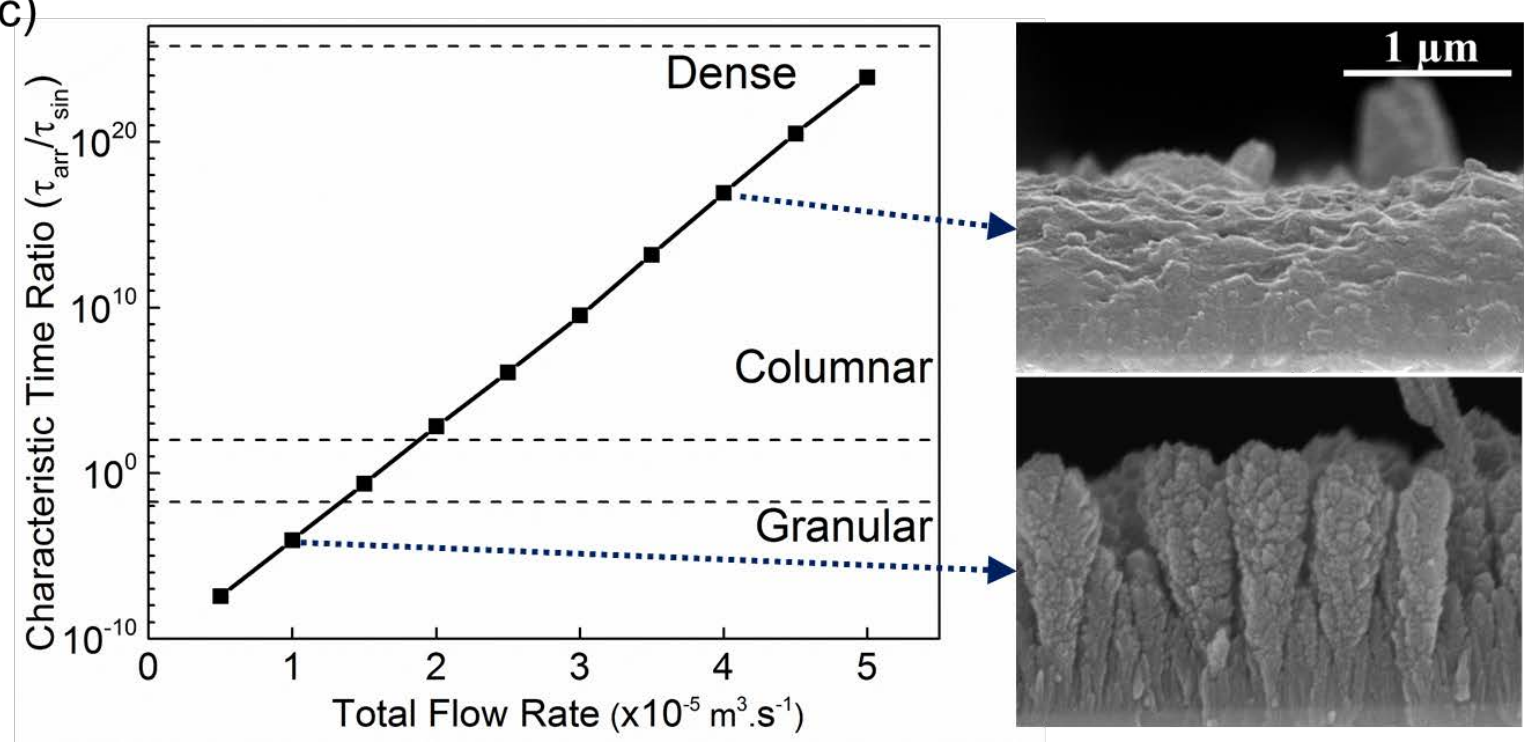

Figure 7: Effect of total inflow flow rate on (a) the particle size distribution, (b) the time averaged temperature, and (c) the characteristic time ratio with the SEM images for the corresponding experimental measurements. 


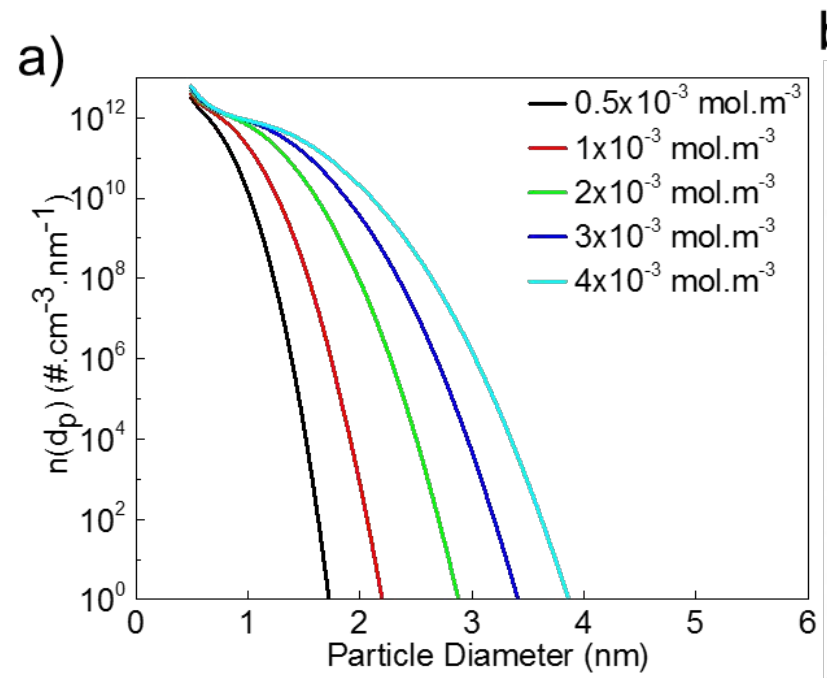

b)

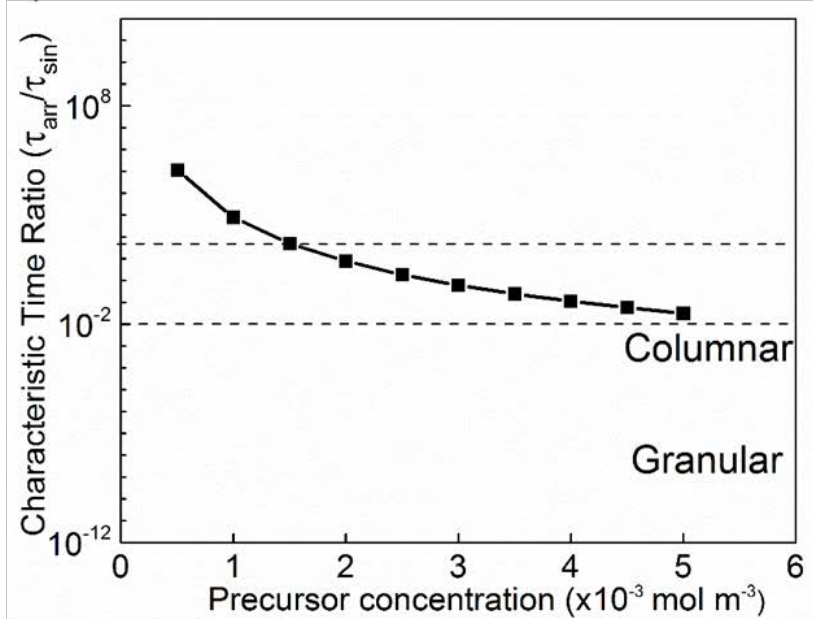

Figure 8: Effect of precursor concentration in the inlet flow on (a) the particle size distribution and (b) the characteristic time ratio 

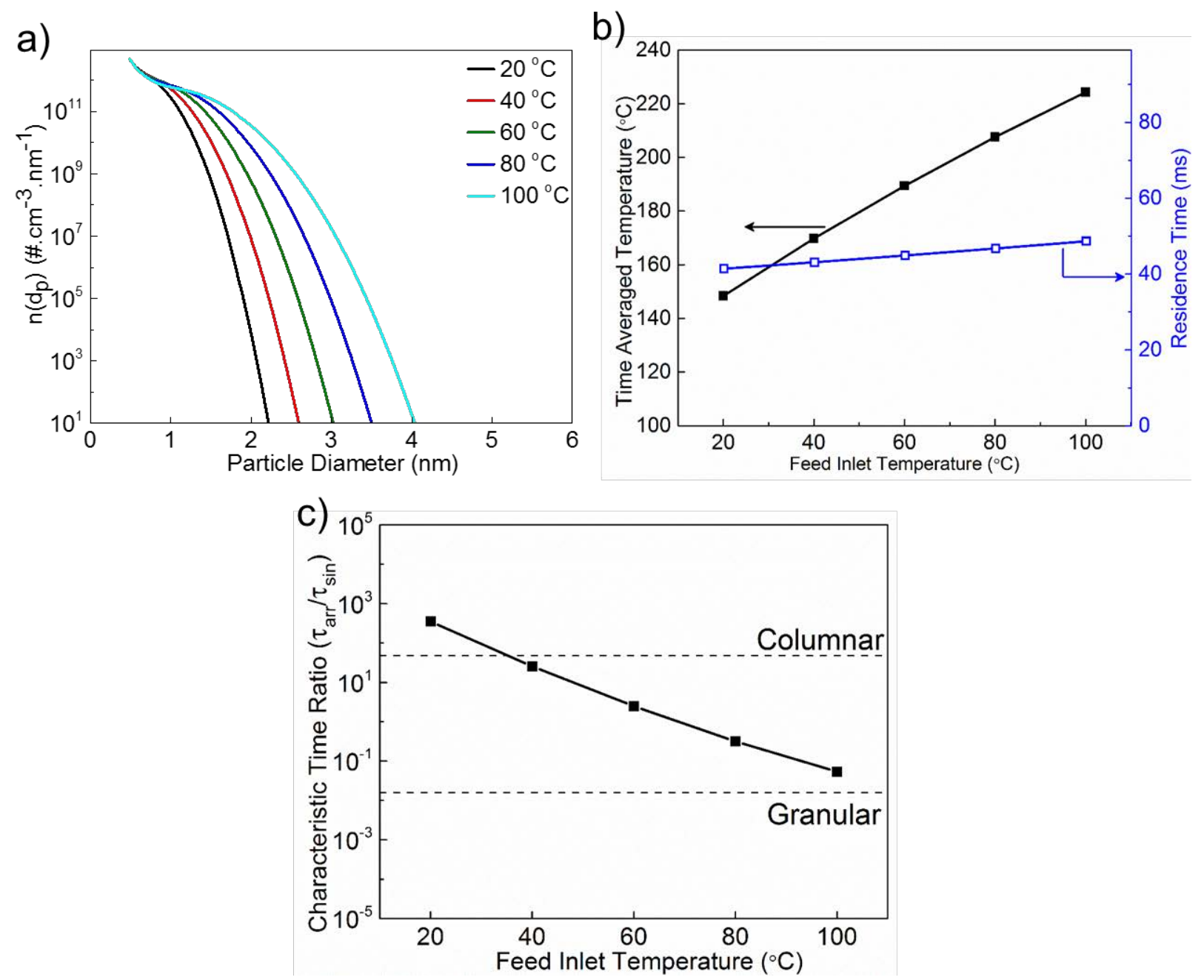

Figure 9: Effect of precursor inlet temperature on (a) the particle size distribution (b) the time averaged temperature and (c) the characteristic time ratio 


\section{Graphical Abstract:}
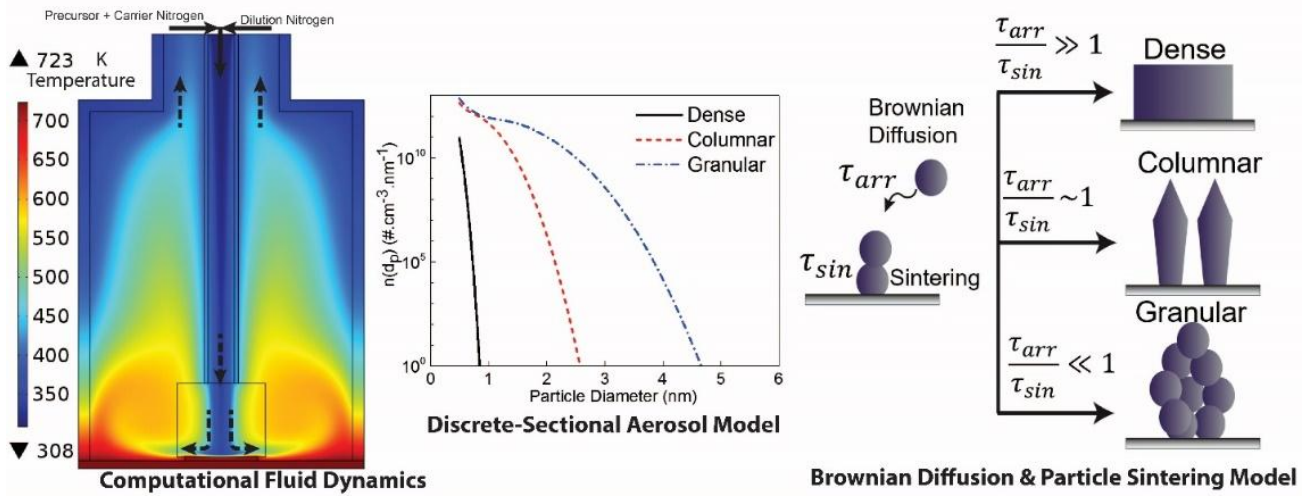